\title{
Prognostic significance of a systemic inflammatory response in patients receiving first- line palliative chemotherapy for recurred or metastatic gastric cancer
}

Jun-Eul Hwang ${ }^{1}$, Ha-Na Kim', Dae-Eun Kim', Hyun-Jung Choi², Sung-Hoon Jung ${ }^{1}$, Hyun-Jeong Shim', Woo-Kyun Bae', Eu-Chang Hwang ${ }^{3}$, Sang-Hee Cho ${ }^{1}$ and Ik-Joo Chung ${ }^{1,4^{*}}$

\begin{abstract}
Background: There is increasing evidence that the presence of an ongoing systemic inflammatory response is associated with poor prognosis in patients with advanced cancers. We evaluated the relationships between clinical status, laboratory factors and progression free survival (PFS), and overall survival (OS) in patients with recurrent or metastatic gastric cancer receiving first-line palliative chemotherapy.

Methods: We reviewed 402 patients with advanced gastric adenocarcinoma who received first-line palliative chemotherapy from June 2004 and December 2009. Various chemotherapy regimens were used. Eastern Cooperative Oncology Group performance status (ECOG PS), C-reactive protein (CRP), albumin, Glasgow prognostic score (GPS), and clinical factors were recorded immediately prior to first-line chemotherapy. Patients with both an elevated CRP ( $>1.0 \mathrm{mg} / \mathrm{dL})$ and hypoalbuminemia $(<3.5 \mathrm{mg} / \mathrm{dL})$ were assigned a GPS of 2. Patients in whom only one of these biochemical abnormalities was present were assigned a GPS of 1, and patients with a normal CRP and albumin were assigned a score of 0 . To evaluate the factors that affected PFS and OS, univariate and multivariate analyses were performed.
\end{abstract}

Results: According to multivariate analysis, the factors independently associated with PFS were ECOG PS (HR 1.37, 95\% Cl 1.02-1.84, $P=0.035$ ), bone metastasis (HR 1.74, 95\% Cl 1.14-2.65, $P=0.009$ ), and CRP elevation (HR 1.64, 95\% $\mathrm{Cl} 1.28-2.09, P=0.001)$. The factors independently associated with OS were ECOG PS (HR 1.33, 95\% Cl 1.01-1.76, $P=$ 0.037), bone metastasis ( $\mathrm{HR} 1.61,95 \% \mathrm{Cl} 1.08-2.39, P=0.017$ ), and $\mathrm{GPS} \geq 1$ (HR 1.76, 95\% Cl 1.41-2.19, $P=0.001$ ).

Conclusions: The results of this study showed that the presence of a systemic inflammatory response as evidenced by the CRP, GPS was significantly associated with shorter PFS and OS in patients with recurrent or metastatic gastric cancer receiving first-line palliative chemotherapy. Bone metastasis and GPS were very useful indicator for survival in patients with recurrent or metastatic gastric cancer receiving palliative chemotherapy.

\section{Background}

Recurred or metastatic gastric cancer has a very poor prognosis, but chemotherapy can improve survival and possibly provide significant palliation of symptoms. Despite the recently reported benefits of chemotherapy, the 5-year survival rate for recurred or metastatic gastric

\footnotetext{
* Correspondence: ijchung@chonnam.ac.kr

'Department of Internal Medicine, Division of Hemato-oncology, Chonnam

National University Medical School, Gwangju, South Korea Full list of author information is available at the end of the article
}

cancer remains at 5-20\% [1-3]. Despite an often short and poor overall survival, there is marked heterogeneity in the duration of survival among patients. Therefore, there have been continuing efforts to investigate the prognostic factors related to survival $[4,5]$.

C-reactive protein (CRP) is an acute phase protein which is synthesized by hepatocytes and;its levels in the serum increase during inflammatory diseases [6]. Cancer growth and resultant invasion induce local tissue damage, disturb local homeostasis, and cause systemic
C Biomed Central 
acute phase responses. Although the exact mechanism by which systemic inflammation arises in cancer patients remains to be clarified, it is generally accepted that cancer associated inflammation is modulated by cancer cells, host stromal cells, and their interactions [7].

There is increasing evidence that the presence of a systemic inflammatory response, as evidenced by elevated concentrations of CRP, is a prognostic factor in patients with advanced cancer [8-10]. High CRP levels are common in patients with advanced disease, because advanced cancer is often associated with an inflammatory response [7].

The Glasgow prognostic score (GPS) was introduced as a useful predictor for survival in patients with cancer by Forrest in 2003, and consists of the combination of 2 values, CRP and albumin $[4,11]$. It has been reported that GPS was associated with prognosis in various types of cancer including non small cell lung cancer, gastric cancer, colorectal cancer, pancreatic cancer, and breast cancer. Nearly all studies evaluated the prognostic value of preoperative CRP in resettable tumors [12]. Clinical factors such as liver metastasis, carcinomatosis peritonei, and bone metastasis are easily identifiable and imply that a patient has an advanced cancer. In this study, we evaluated the relationships between carcinomatosis peritonei, liver metastasis, bone metastasis, ECOG PS, albumin, CRP, GPS, and progression free survival (PFS), and overall survival (OS) in patients with recurrent or metastatic gastric cancer receiving first-line palliative chemotherapy.

\section{Methods \\ Patients}

We evaluated patients with advanced gastric cancer who had received palliative chemotherapy between June 2004 and December 2009 at Chonnam National University Hwasun Hospital (Gwangju, Korea). Patients were staged using a combination of endoscopy, computed tomography (CT) scan of the chest and abdomen, and additionally, positron emission tomography or bone scan when clinically indicated.

The criteria for case inclusion were as follows: (1) histologically confirmed gastric adenocarcinoma, (2) no prior chemotherapy or radiotherapy except for adjuvant treatment, (3) presence of metastatic disease, and (4) availability of clinical data at the initiation of therapy and follow-up. Of the 532 patients screened, 402 fulfilled the inclusion criteria and were enrolled in this retrospective analysis.

ECOG PS was evaluated according to the Eastern Cooperative Oncology Group criteria. The clinical tumor response was assessed radiologically by CT scanning after every 2 or 3 courses of chemotherapy according to the Response Evaluation Criteria in Solid Tumors
(RECIST version 1.0) and clinically based on control of symptoms

Chemotherapy regimens had included a variety of agents such as taxane, irinotecan, cisplatin, oxaliplatin, 5-FU, S-1, and capecitabine. Oral fluoropyrimidines such as S-1 and capecitabine are replacing infusional 5FU, and doublet chemotherapy regimens were most commonly used.

This study was approved by the institutional review board of Chonnam National University Medical School Research Institution (2011-109).

\section{Measurement of serum CRP}

Routine laboratory measurements of CRP and albumin were carried out one day before the first cycle of chemotherapy. The limit of detection of the CRP assay was $<0.03 \mathrm{mg} / \mathrm{dL}$, with the upper limit of normal values being $<1.0 \mathrm{mg} / \mathrm{dL}$. Serum CRP was measured by latex turbidimetric immunoassay using a HITACHI 7600 (Hitachi, Tokyo, Japan). The coefficients of variation for these methods, over the range of measurements, were < $5 \%$, as established by routine quality control.

The GPS was derived as previously described [4,11]. Patients with both an elevated CRP $(>1.0 \mathrm{mg} / \mathrm{dL})$ and hypoalbuminemia $(<3.5 \mathrm{mg} / \mathrm{dL})$ were assigned a score of 2. Patients in whom only one of these biochemical abnormalities was present were assigned of 1 . Patients in whom neither of these abnormalities was present were assigned a score of 0 .

\section{Statistics}

The SPSS software package, version 17.0 (SPSS Inc, Chicago, IL, USA) was used for statistical analysis. The Kaplan-Meier analysis was applied to assess factors affecting overall survival (OS) and progression free survival (PFS): (1) OS was defined as the time from the first date of the first-line chemotherapy or to death from any cause or the last follow-up visit (2) PFS was defined as the time from the first date of the first-line chemotherapy to disease progression. Factors included in the univariate analysis using Kaplan-Meier methods were age, gender, liver metastasis, peritoneal metastasis, bone metastasis, albumin, CRP, ECOG PS, and GPS. Among the factors, those with $P<0.2$ were selected and included in the multivariate regression analysis using the Cox proportional hazards regression model, which was performed to achieve an adjusted hazard ratio (HR) to determine prognostic factors for OS and PFS. $P<$ 0.05 was considered significant for all analyses.

\section{Results}

This study included a total of 402 patients who received first-line chemotherapy in the Department of Oncology at Chonnam National University Hwasun Hospital 
(Gwangju, Republic of Korea). We collected follow-up patient data from the cancer registry. All data were prospectively recorded and only the survival data was updated at the time of analyses. The median follow-up time was 11.4 months and ranged from 1.1 months to 58.5 months. The baseline clinical characteristics of the patients at the start of first-line chemotherapy are shown in Table 1 and Table 2. The median age of the patients was 59 years, with a range of $19-80$ years. A total of 203 patients $(72.9 \%)$ were male, and 73 patients (48.2\%) had an ECOG PS of 2-3. A total of 125 patients (31.1\%) had liver metastases, and 159 patients (39.6\%), and 28 patients $(7.0 \%)$ had peritoneal metastases and bone metastases, respectively. A total of 140 patients (34.9\%) had an elevated CRP concentration (>1 mg/dL),

Table 1 Univariate analysis of clinical factors and GPS for progression free survival $(n=402)$

\begin{tabular}{llll}
\hline & Patients (\%) & mPFS months (95\% Cl) & P-value \\
\hline Age & & & \\
\hline$<60$ & $203(50.5)$ & $4.3(3.8-4.7)$ & 0.840 \\
\hline$\geq 60$ & $199(49.5)$ & $4.5(3.9-5.1)$ & \\
\hline Gender & & & \\
\hline Male & $293(72.9)$ & $4.5(3.9-5.0)$ & 0.381 \\
\hline Female & $109(27.1)$ & $4.3(3.6-4.9)$ & \\
\hline Liver Mets & & & 0.243 \\
\hline Yes & $125(31.1)$ & $4.1(3.6-4.5)$ &
\end{tabular}

Peritoneal Mets

\begin{tabular}{llll}
\hline Yes & $159(39.6)$ & $4.3(3.8-4.8)$ & 0.128 \\
\cline { 1 - 2 } No & $243(60.4)$ & $4.6(3.9-5.2)$ & \\
\hline
\end{tabular}

\begin{tabular}{|c|c|c|c|}
\hline \multicolumn{4}{|c|}{ Bone Mets } \\
\hline Yes & $28(7.0)$ & $3.9(2.4-5.3)$ & 0.032 \\
\hline No & $374(93.0)$ & $4.5(4.0-5.0)$ & \\
\hline \multicolumn{4}{|c|}{ Albumin } \\
\hline$<3.5$ & 77 (19.2) & $3.4(2.2-4.5)$ & 0.013 \\
\hline$\geq 3.5$ & $325(80.8)$ & $4.6(4.1-5.2)$ & \\
\hline \multicolumn{4}{|l|}{ CRP } \\
\hline$\leq 1$ & $262(65.1)$ & $5.3(4.5-6.0)$ & 0.001 \\
\hline$>1$ & $140(34.9)$ & $3.4(2.7-4.1)$ & \\
\hline \multicolumn{4}{|c|}{ ECOG PS } \\
\hline $0-1$ & $329(81.8)$ & $4.6(4.0-5.1)$ & 0.002 \\
\hline$\geq 2$ & $73(48.2)$ & $3.0(2.0-3.9)$ & \\
\hline \multicolumn{4}{|l|}{ GPS } \\
\hline 0 & $238(59.2)$ & $5.5(4.8-6.2)$ & 0.001 \\
\hline 1 & $111(27.6)$ & $3.4(2.7-4.1)$ & \\
\hline 2 & $53(13.2)$ & $3.3(2.0-4.7)$ & \\
\hline
\end{tabular}

mPFS: median progression free survival, Mets: Metastasis, CRP: C-reactive protein, ECOG PS: Eastern Cooperative Oncology Group, performance status, GPS: Glascow prognostic score
Table 2 Univariate analysis of clinical factors and GPS for overall survival $(n=402)$

\begin{tabular}{|c|c|c|c|}
\hline & Patients (\%) & mOS months $(95 \% \mathrm{Cl})$ & $P$-value \\
\hline \multicolumn{4}{|l|}{ Age } \\
\hline$<60$ & $203(50.5)$ & $11.6(10.1-13.2)$ & 0.808 \\
\hline$\geq 60$ & $199(49.5)$ & $12.0(9.9-14.1)$ & \\
\hline \multicolumn{4}{|l|}{ Gender } \\
\hline Male & $293(72.9)$ & $11.7(10.1-13.3)$ & 0.952 \\
\hline Female & $109(27.1)$ & $12.1(10.6-13.6)$ & \\
\hline \multicolumn{4}{|c|}{ Liver Mets } \\
\hline Yes & $125(31.1)$ & $11.4(8.5-14.3)$ & 0.352 \\
\hline No & $277(68.9)$ & $12.0(10.7-13.3)$ & \\
\hline
\end{tabular}

Peritoneal Mets

\begin{tabular}{llll}
\hline Yes & $159(39.6)$ & $9.9(8.4-11.5)$ & 0.023 \\
\cline { 1 - 2 } No & $243(60.4)$ & $13.0(11.2-14.8)$ & \\
\hline Bone Mets & & & \\
\hline Yes & $28(7.0)$ & $8.2(7.1-9.3)$ & 0.117 \\
\cline { 1 - 2 } No & $374(93.0)$ & $12.1(10.9-13.2)$ &
\end{tabular}

\begin{tabular}{|c|c|c|c|}
\hline \multicolumn{4}{|c|}{ Albumin } \\
\hline$<3.5$ & $77(19.2)$ & $7.7(5.9-9.5)$ & 0.001 \\
\hline$\geq 3.5$ & $325(80.8)$ & $12.4(10.7-14.2)$ & \\
\hline \multicolumn{4}{|l|}{$\overline{C R P}$} \\
\hline$\leq 1$ & $262(64.9)$ & $14.8(12.7-17.0)$ & 0.001 \\
\hline$>1$ & $140(34.8)$ & $8.7(7.3-10.2)$ & \\
\hline \multicolumn{4}{|c|}{ ECOG PS } \\
\hline $0-1$ & $329(81.8)$ & $12.4(10.9-13.8)$ & 0.003 \\
\hline$\geq 2$ & $73(48.2)$ & $8.7(7.3-10.0)$ & \\
\hline \multicolumn{4}{|l|}{ GPS } \\
\hline 0 & $238(59.2)$ & $15.3(13.1-17.5)$ & 0.001 \\
\hline 1 & $111(27.6)$ & $8.9(7.2-10.7)$ & \\
\hline 2 & $53(13.2)$ & $7.6(4.9-10.2)$ & \\
\hline
\end{tabular}

mOS: median overall survival, Mets: metastasis, CRP: C-reactive protein, ECOG PS: Eastern Cooperative Oncology Group, performance status, GPS: Glascow prognostic score

and 77 patients $(19.2 \%)$ had hypoalbuminemia $(<3.5$ $\mathrm{mg} / \mathrm{dL})$.

The most commonly used first-line chemotherapy regimens were taxanes and cisplatin $(n=191,47.5 \%)$. The specific chemotherapy regimens are shown in Table 3. A complete response to first-line chemotherapy was achieved in 25 patients $(6.2 \%)$, and a partial response was achieved in 120 patients (29.9\%), giving an overall response rate of $36.1 \%$ and a disease control rate of $70.9 \%$. The results of chemotherapy are shown in Table 4 . The median progression free survival was 4.4 months (95\% CI 4.018-4.782), and the median overall survival was 11.8 months $(95 \% \mathrm{CI}$ 10.727-12.873).

The univariate analysis demonstrated that 5 clinical factors were significantly associated with a shorter PFS; these factors included bone metastases, albumin $<3.5$ 
Table 3 Regimens of chemotherapy

\begin{tabular}{lll}
\hline Regimen & $\begin{array}{l}\text { No. of } \\
\text { patients }\end{array}$ & $\%$ \\
\hline Taxane/Cisplatin & 191 & 47.5 \\
\hline Taxane/Cisplatin/5-FU & 94 & 23.4 \\
\hline Irinotecan/5-FU & 9 & 2.2 \\
\hline Oxaliplatin/5-FU & 52 & 12.9 \\
\hline 5-FU (oral or infusional fluoropyrimidines)/ & 21 & 5.2 \\
Cisplatin & & \\
\hline Oral fluoropyrimidine & 35 & 8.7 \\
\hline Total & 402 & 100.0 \\
\hline
\end{tabular}

$\mathrm{mg} / \mathrm{dL}, \mathrm{CRP}>1 \mathrm{mg} / \mathrm{dL}, \mathrm{PS} \geq 2$, GPS $\geq 1$ (Table 1 ). The univariate analysis also demonstrated that 5 clinical factors were significantly associated with a shorter OS; these factors included peritoneal metastasis, albumin < $3.5 \mathrm{mg} / \mathrm{dL}, \mathrm{CRP}>1 \mathrm{mg} / \mathrm{dL}, \mathrm{PS} \geq 2$, and GPS $\geq 1$ (Table 2).

Cox multivariate analysis for PFS and OS was performed. Among the clinical factors, those with $P<0.2$ were selected and included in the multivariate regression analysis using the Cox proportional hazards regression model. The result of the analysis identified the independent prognostic factors for PFS and OS. The independent poor prognostic factors for PFS were PS $\geq$ 2 (HR 1.37, 95\% CI 1.02-1.84; $P=0.035$ ), CPR $>1 \mathrm{mg} /$ dL (HR 1.64 95\% CI 1.28-2.09; $P=0.001$ ), and bone metastasis (HR 1.74, 95\% CI 1.14-2.65; $P=0.009$ ). The independent prognostic factors for OS were PS $\geq 2$ (HR 1.33, 95\% CI 1.01-1.76; $P=0.037$ ), GPS 1 (HR 1.75, $95 \%$ CI $1.37-2.26$; $P=0.001$ ), GPS 2 (HR $1.79,95 \%$ CI 1.29 2.47; $P=0.001$ ), and bone metastasis (HR $1.6195 \% \mathrm{CI}$ 1.08 -2.39; $P=0.017$ ) (Table 5). Figure 1 shows the survival curve for patients according to GPS.

In this study, GPS 1 was most commonly found to be a result of an elevated CRP (87 of 111, 78.4\%). Among the patients with GPS 1, the patients with increased CRP and normal albumin level had a better median

Table 4 Response to chemotherapy $(n=402)$

\begin{tabular}{lll}
\hline Response & No of Patient & \% \\
\hline$C R$ & 25 & 6.2 \\
\hline$P R$ & 120 & 29.9 \\
\hline$S D$ & 140 & 34.8 \\
\hline$P D$ & 103 & 25.6 \\
\hline ORR (CR + PR) & 145 & 36.1 \\
\hline DCR (CR + PR + SD) & 285 & 70.9 \\
\hline NE & 14 & 3.5 \\
\hline Total & 402 & 100.0
\end{tabular}

CR: complete response, PR: partial response, SD: stable disease, PD: progressive disease, ORR: overall response rate, DCR: disease control rate, NE: not evaluable
Table 5 Progression free survival and overall survival in advanced gastric cancer patients receiving first-line chemotherapy (multivariate analysis)

\begin{tabular}{lll}
\hline \multirow{2}{*}{ Factors } & \multicolumn{2}{l}{ Progression free survival } \\
\cline { 2 - 3 } & Hazard ratio $(95 \% \mathrm{Cl})$ & $\boldsymbol{P}$-value \\
\hline ECOG PS $(\geq 2)$ & $1.37(1.02-1.84)$ & 0.035 \\
\hline CRP $(>1)$ & $1.64(1.28-2.09)$ & 0.001 \\
\hline Bone metastasis & $1.74(1.14-2.65)$ & 0.009 \\
\hline & Overall survival & \\
\cline { 2 - 3 } & Hazard ratio $(95 \% \mathrm{Cl})$ & $P$-value \\
\hline ECOG PS $(\geq 2)$ & $1.33(1.01-1.76)$ & 0.037 \\
\hline GPS $(1)$ & $1.75(1.37-2.26)$ & 0.001 \\
\hline GPS $(2)$ & $1.79(1.29-2.47)$ & 0.001 \\
\hline Bone metastasis & $1.61(1.08-2.39)$ & 0.017 \\
\hline
\end{tabular}

ECOG PS: Eastern Cooperative Oncology Group, performance status, CRP: Creactive protein

progression survival and median overall survival than the patients with normal CRP and decreased albumin level; however, the difference was not statistically significant (Table 6).

\section{Discussion}

CRP is a nonspecific but sensitive marker of systemic inflammatory response, and might be expressed in selected tumor cells [13]. The biological basis for the correlation between elevations in this general marker of systemic inflammation and disease risk and outcome are not completely understood. The liberation of multiple proinflammatory cytokines, including interleukin-1 (IL-1), IL-6, and tumor necrosis factor- $\alpha$ from the tumor environment eventually results in the induction of CRP synthesis from the liver and other tissues [14]. Based on many recent studies, it is now widely accepted that an elevated CRP value is a reliable indicator of poor prognosis for certain malignant tumors including melanoma, non-Hodgkin's lymphoma, colorectal, lung, prostate and ovary $[12,15,16]$.

Several studies demonstrated that low albumin concentrations are significantly associated with poorer survival in patients with gastric cancer $[17,18]$. In patients with gastric cancer, it is recognized that there may be reduced caloric intake due to stenosis of the cardia or pylorus. However, several recent reports showed that the systemic inflammatory response plays a major role in the progressive nutritional and functional decline of patients with cancer [19]. Indeed, measurement of the systemic inflammatory response, particularly CRP, has been included in the definition of cancer cachexia, together with weight loss and reduced calorie intake [20]. It thus appears that the development of hypoalbuminemia is secondary to an ongoing systemic inflammatory response and poor cancer specific survival is secondary to the systemic inflammatory response in 


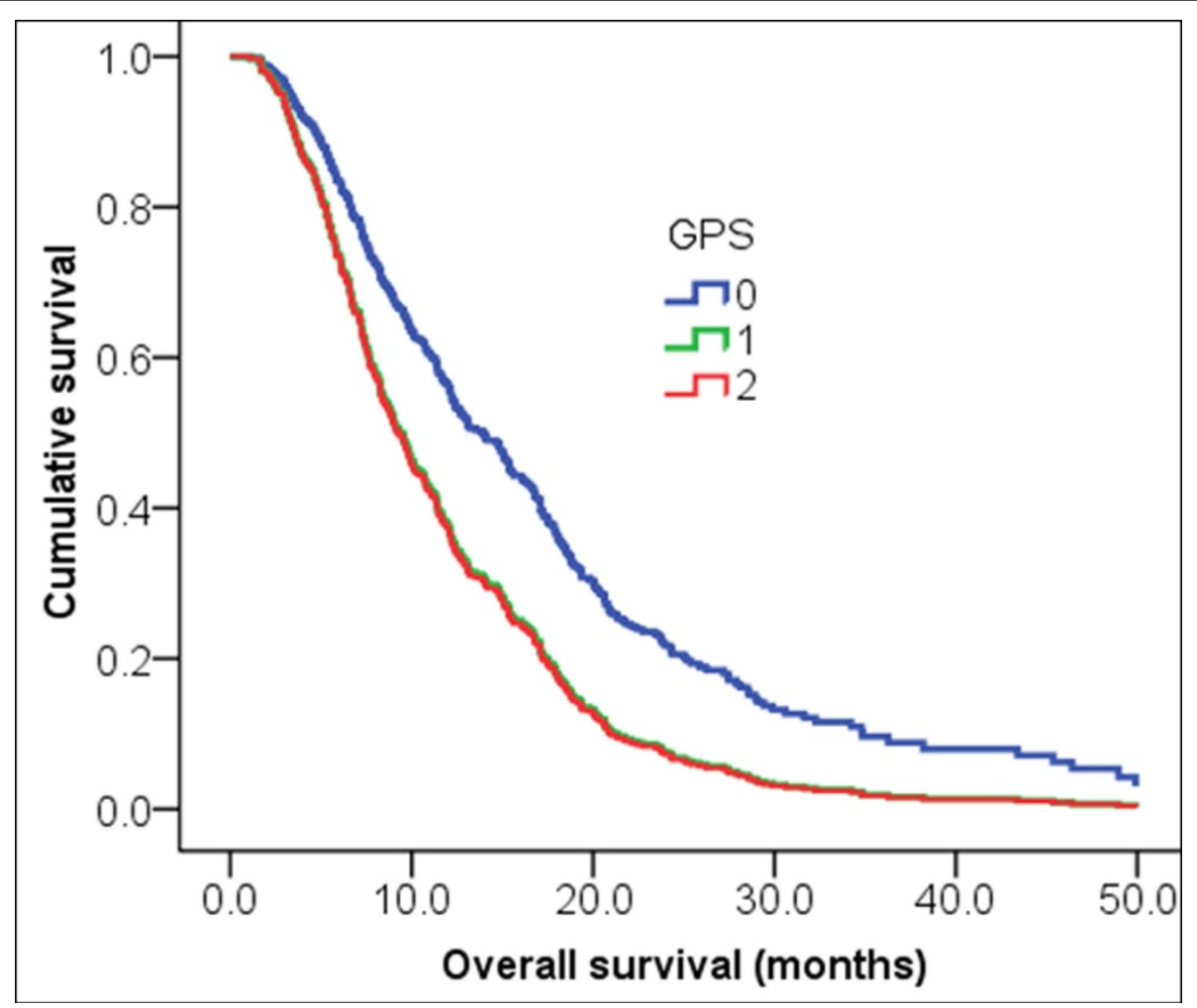

Figure 1 GPS was an independent poor prognostic factor for OS in recurred or metastatic gastric cancer patients receiving first-line palliative chemotherapy (GPS $=1$, HR $1.7595 \% \mathrm{Cl} 1.37-2.26, P=0.001$; GPS $=2$, HR 1.79, $95 \% \mathrm{Cl} 1.29-2.47, P=0.001$ ).

patients with gastric cancer [21]. GPS can thus reflect both the presence of a systemic inflammatory response and the progressive nutritional decline of the patient with advanced cancer. In this study, GPS 1 was most commonly associated with increased CRP (87 of 111, $78.4 \%)$. Our results also demonstrated that increased CRP was associated with shorter PFS, and a GPS $\geq 1$ was associated with shorter OS in advanced gastric cancer in palliative chemotherapy settings.

Table 6 Patients with increased CRP and normal albumin level had a better median progression free survival and median overall survival than patients with normal CRP and decreased albumin level in the patients with GPS 1 (not statistically significant)

\begin{tabular}{|c|c|c|c|}
\hline & $\begin{array}{l}\text { Patients with increased } \\
\text { CRP and normal } \\
\text { albumin level }(n=87)\end{array}$ & $\begin{array}{l}\text { Patients with normal } \\
\text { CRP and decreased } \\
\text { albumin level }(n=24)\end{array}$ & $P$-value \\
\hline \multirow{2}{*}{$\begin{array}{l}\text { mPFS } \\
\text { (months) }\end{array}$} & 3.433 & 2.533 & 0.489 \\
\hline & (95\% Cl 2.648-4.219) & (95\% Cl 0.000-5.284) & \\
\hline \multirow{2}{*}{$\begin{array}{l}\text { mOS } \\
\text { (months) }\end{array}$} & 8.9 & 8.7 & 0.915 \\
\hline & $(95 \%$ Cl 7.003-10.930) & $(95 \%$ Cl 5.779-11.621) & \\
\hline
\end{tabular}

mPFS: median progression free survival, mOS: median overall survival, CRP: Creactive protein
In gastric cancers, almost all studies evaluated the prognostic value of the preoperative CRP in resettable tumors [12]. In a recent study of 204 patients who underwent curative resection of gastric carcinoma, preoperative CRP elevations were found to be independently predictive of shorter overall survival [22]. To our knowledge, only 2 studies demonstrated the relationship between GPS and cancer specific survival in unresectable gastric cancer $[4,5]$. These studies showed that GPS was a significant independent predictor of cancer survival in unresectable gastroesophageal cancer. However, these studies also included esophageal cancer and diverse treatment modalities such as chemotherapy, radiotherapy, and endoscopic laser were used for palliative treatment. In our study, we only enrolled patients receiving first-line palliative chemotherapy for gastric adenocarcinoma. We excluded gastroesophageal junction cancer, because the underlying pathophysiology of cancers of the gastroesophageal junction and the corresponding therapeutic approach differ substantially from those for gastric cancer proper [23].

Although gastric cancer is thought to spread more commonly to the peritoneum or liver than to bone, the reported incidence of skeletal lesions varies between 1 and $45 \%$ [24]. The presence of bone metastasis has 
consistently been associated with poor prognosis in studies of Korean gastric cancer patients. In their report analyzing 1,455 metastatic gastric cancer patients who received first-line chemotherapy, Lee et al. reported that the presence of bone metastasis was an independent poor prognostic factor [18]. In our study, bone metastasis was also independently associated with poor prognosis (median OS 8.2 vs. 12.1 months, bone metastasis (+) vs. (-), $P=0.017)$.

The mechanism by which a systemic inflammatory response might influence cancer survival is not clear. It may be that an elevated systemic inflammatory response is associated with a poor local immune response to the tumor and therefore increased lymph node spread and metastasis [21]. Also, the presence of a systemic inflammatory response and the associated nutritional decline may influences tolerance and compliance with active treatment $[25,26]$. Andreyev et al. reported that the poorer outcome of chemotherapy in advanced gastrointestinal cancer patients with weight loss appears to be as a result of receiving less chemotherapy due to toxicity rather than poorer tumor response [27]. In this study, among the patients with GPS 1 , the patients with normal CRP and decreased albumin level $(n=24)$ had a tendency to have a poorer median progression free survival and median overall survival than the patients with increased CRP and normal albumin level $(n=87)$. In this group of the patients, decreased albumin level might be associated with the chronic nutritional decline and poor ECOG PS.

\section{Conclusions}

The presence of a systemic inflammatory response as evidenced by the CRP and GPS, appears to be a useful indicator of prognosis in patients receiving first-line chemotherapy for metastatic gastric cancer. Poor performance status and bone metastasis were also associated with poor prognosis. CRP, GPS, and bone metastasis are easily identifiable and objective clinical parameters. These parameters could facilitate the individual patient risk assessment and thus, a more appropriate prediction of survival for each patient.

\footnotetext{
Author details

'Department of Internal Medicine, Division of Hemato-oncology, Chonnam National University Medical School, Gwangju, South Korea. ${ }^{2}$ Department of Laboratory Medicine, Chonnam National University Medical School, Gwangju, South Korea. ${ }^{3}$ Department of Urology, Chonnam National University Medical School, Gwangju, South Korea. ${ }^{4}$ Department of Internal Medicine, Chonnam National University Hwasun Hospital, Jeollanamdo 519-809, South Korea.

\section{Authors' contributions}

JEH is a main author. DEK, WKB, HJS, SHJ and SHC performed the chemotherapy for patients and revised the manuscript. ECH made special contributions to the statistical analysis. IJC conceived of the study, and approved the final manuscript. All authors read and approved the final manuscript.
}

\section{Competing interests}

The authors declare that they have no competing interests.

Received: 7 July 2011 Accepted: 21 November 2011

Published: 21 November 2011

\section{References}

1. Bang YJ, Van Cutsem E, Feyereislova A, chung HC, Shen L, Sawaki A, Lordick F, Ohtsu A, Omuro Y, Satoh T, Aprile G, Kulikov E, Hill J, Lehle M, Rüschoff J, Kang YK, ToGA Trial Investigators: Trastuzumab in combination with chemotherapy versus chemotherapy alone for treatment of HER2positive advanced gastric or gastro-oesophageal junction cancer (ToGA): a phase 3, open-label, randomised controlled trial. Lancet 2010, 376:687-697.

2. Koizumi W, Narahara H, Hara T, Takagane A, Akiya T, Takagi M, Miyashita K, Nishizaki T, Kobayashi O, Takiyama W, Toh Y, Nagaie T, Takagi S, Yamamura Y, Yanaoka K, Orita H, Takeuchi M: S-1 plus cisplatin versus S-1 alone for first-line treatment of advanced gastric cancer (SPIRITS trial): a phase III trial. Lancet Oncol 2008, 9:215-221.

3. Van Cutsem E, Moiseyenko VM, Tjulandin S, Majlis A, Constenla M, Boni C, Rodrigues A, Fodor M, Chao Y, Voznyi E, Risse ML, Ajani JA, V325 study Group: Phase III study of docetaxel and cisplatin plus fluorouracil compared with cisplatin and fluorouracil as first-line therapy for advanced gastric cancer: a report of the V325 Study Group. J Clin Oncol 2006, 24:4991-4997.

4. Crumley AB, McMillan DC, McKernan M, McDonald AC, Stuart RC: Evaluation of an inflammation-based prognostic score in patients with inoperable gastro-oesophageal cancer. Br J Cancer 2006, 94:637-641.

5. Crumley AB, Stuart RC, McKernan M, McDonald AC, McMillan DC: Comparison of an inflammation-based prognostic score (GPS) with performance status (ECOG-ps) in patients receiving palliative chemotherapy for gastroesophageal cancer. J Gastroenterol Hepatol 2008, 23:e325-e329.

6. Zimmerman MA, Selzman CH, Cothren C, Sorensen AC, Raeburn CD, Harken AH: Diagnostic implications of C-reactive protein. Arch Surg 2003, 138:220-224.

7. Yamashita $\mathrm{H}$, Katai $\mathrm{H}$ : Systemic inflammatory response in gastric cancer. World J Surg 2010, 34:2399-2400.

8. O'Gorman P, McMillan DC, McArdle CS: Prognostic factors in advanced gastrointestinal cancer patients with weight loss. Nutr Cancer 2000, 37:36-40.

9. Scott HR, McMillan DC, Forrest LM, Brown DJ, McArdle CS, Milroy R: The systemic inflammatory response, weight loss, performance status and survival in patients with inoperable non-small cell lung cancer. $\mathrm{Br} J$ Cancer 2002, 87:264-267.

10. Maltoni M, Caraceni A, Brunelli C, Broeckaert B, Christakis N, Eychmueller S, Glare P, Nabal M, Vigano A, Larkin P, De Conno F, Hanks G, Kaasa S, Steering Committee of the European Association for Palliative Care: Prognostic factors in advanced cancer patients: evidence-based clinical recommendations-a study by the Steering Committee of the European Association for Palliative Care. J Clin Oncol 2005, 23:6240-6248.

11. Forrest LM, McMillan DC, MCArdle CS, Angerson WJ, Dunlop DJ: Evaluation of cumulative prognostic scores based on the systemic inflammatory response in patients with inoperable non-small-cell lung cancer. $\mathrm{Br} J$ Cancer 2003, 89:1028-1030.

12. Roxburgh CS, McMillan DC: Role of systemic inflammatory response in predicting survival in patients with primary operable cancer. Future Oncol 2010, 6:149-163.

13. Nozoe T, Korenaga D, Futatsugi M, Saeki H, Maehara $Y$, Sugimachi $K$. Immunohistochemical expression of C-reactive protein in squamous cell carcinoma of the esophagus - significance as a tumor marker. Cancer Lett 2003, 192:89-95.

14. Du Clos TW: Function of C-reactive protein. Ann Med 2000, 32:274-278.

15. Legouffe $E$, Rodriguez C, Picot MC, Richard B, Klein B, Rossi JF, Commes T: C-reactive protein serum level is a valuable and simple prognostic marker in non Hodgkin's lymphoma. Leuk Lymphoma 1998, 31:351-357.

16. Tartour E, Dorval T, Mosseri V, Deneux L, Mathiot C, Brailly H, Montero F, Joyeux I, Pouillart P, Fridman WH: Serum interleukin 6 and C-reactive protein levels correlate with resistance to IL-2 therapy and poor survival in melanoma patients. Br J Cancer 1994, 69:911-913. 
17. Lien YC, Hsieh CC, Wu YC, Hsu HS, Hsu WH, Wang LS, Huang MH,

Huang BS: Preoperative serum albumin level is a prognostic indicator for adenocarcinoma of the gastric cardia. J Gastrointest Surg 2004, 8:1041-1048.

18. Lee J, Lim T, Uhm JE, Park KW, Park SH, Lee SC, Park JO, Park YS, Lim HY, Sohn TS, Noh JH, Heo JS, Park CK, Kim S, Kang WK: Prognostic model to predict survival following first-line chemotherapy in patients with metastatic gastric adenocarcinoma. Ann Oncol 2007, 18:886-891.

19. McMillan DC: An inflammation-based prognostic score and its role in the nutrition-based management of patients with cancer. Proc Nutr Soc 2008, 67:257-262.

20. Fearon KC, Voss AC, Hustead DS: Definition of cancer cachexia: effect of weight loss, reduced food intake, and systemic inflammation on functional status and prognosis. Am J Clin Nutr 2006, 83:1345-1350

21. Crumley AB, Stuart RC, MCKernan M, MCMillan DC: Is hypoalbuminemia an independent prognostic factor in patients with gastric cancer? World J Surg 2010, 34:2393-2398.

22. Nozoe T, Iguchi T, Adachi E, Matsukuma A, Ezaki T: Preoperative elevation of serum C-reactive protein as an independent prognostic indicator for gastric cancer. Surg Today 2011, 41:510-513.

23. Tepper JE: Is radiation therapy needed in the treatment of gastroesophageal junction adenocarcinoma? Gastrointest Cancer Res 2008, 2:S2-S5.

24. Park HS, Rha SY, Kim HS, Hyung WJ, Park JS, Chung HC, Noh SH, Jeung HC: A Prognostic Model to Predict Clinical Outcome in Gastric Cancer Patients with Bone Metastasis. Oncology 2011, 80:142-150.

25. McMillan DC, Scott HR, Watson WS, Preston T, Milroy R, McArdle CS Longitudinal study of body cell mass depletion and the inflammatory response in cancer patients. Nutr Cancer 1998, 31:101-105.

26. Bromwich E, McMillan DC, Lamb GW, Vasey PA, Aitchison M: The systemic inflammatory response, performance status and survival in patients undergoing alpha-interferon treatment for advanced renal cancer. $\mathrm{Br} J$ Cancer 2004, 91:1236-1238.

27. Andreyev HJ, Norman AR, Oates J, Cunningham D: Why do patients with weight loss have a worse outcome when undergoing chemotherapy for gastrointestinal malignancies? Eur J Cancer 1998, 34:503-509.

\section{Pre-publication history}

The pre-publication history for this paper can be accessed here: http://www.biomedcentral.com/1471-2407/11/489/prepub

doi:10.1186/1471-2407-11-489

Cite this article as: Hwang et al:: Prognostic significance of a systemic inflammatory response in patients receiving first-line palliative chemotherapy for recurred or metastatic gastric cancer. BMC Cancer 2011 11:489.

\section{Submit your next manuscript to BioMed Central and take full advantage of:}

- Convenient online submission

- Thorough peer review

- No space constraints or color figure charges

- Immediate publication on acceptance

- Inclusion in PubMed, CAS, Scopus and Google Scholar

- Research which is freely available for redistribution 